\title{
Endoscopic Endonasal Management of Extraskeletal Sinonasal Ewing's Sarcoma
}

\author{
Anuragini Gupta ${ }^{1}$, Sushmita Gupta ${ }^{2}$, Deepika Chaudhary ${ }^{3}$, Anish Gupta ${ }^{4}$, Ashok K Gupta ${ }^{5}$
}

\begin{abstract}
Ewing's sarcoma (ES) is a primary neoplasm of the skeletal system. Ewing's sarcoma of the head-and-neck region constitutes about 1-4\% of extraskeletal Ewing's sarcoma (EES cases). Sinonasal EES is extremely rare and there is limited literature on this entity. Establishing the diagnosis requires a histopathological examination, immunohistochemistry, and a cytogenetic analysis along with a CT scan and MRI of the paranasal sinuses. They require multimodality treatment including surgical resection followed by chemotherapy and/or radiotherapy. We present a case report of a 31-year-old male patient presenting with a right-sided headache associated with nausea and vomiting, right nasal obstruction, a mass protruding from the right nostril and associated with occasional nasal bleed for 4 months with aggravation of symptoms since last 15 days along with proptosis of the right eye. On nasal examination, reddish-brown friable slough covered mass protruding from the right nostril bleeding on manipulation. Endoscopic endonasal excision biopsy revealed a round cell tumor-ES/primitive neuroectodermal tumor (PNET). The patient received radiotherapy and chemotherapy postoperatively and is on regular follow-up for 2 years and is disease-free.

Keywords: Endoscopic, Endoscopic excision, Rare.

Clinical Rhinology An International Journal (2020): 10.5005/jp-journals-10013-1370
\end{abstract}

\section{INTRODUCTION}

Ewing's sarcoma (ES) is a primary neoplasm of the skeletal system. It broadly includes a family of tumors behaving similarly and is morphologically, histologically similar requiring special markers for differentiation, better known as the Ewing's sarcoma family of tumors (ESFT). Ewing's sarcoma family of tumors encompasses a group of highly aggressive malignant neoplasms sharing a common spontaneous genetic translocation that affects mostly children and young adults. ${ }^{1}$ About $80 \%$ of patients are younger than 20 years of age with the highest incidence in the second decade of life. ${ }^{1-3}$ Ewing's sarcoma is more common in white populations and has a slight male predominance. ${ }^{1,4,5}$ They have many overlapping features suggesting a common histogenesis. Ewing's sarcoma family of tumors includes peripheral primitive neuroectodermal tumor (PNET), neuroepithelioma, and Askin tumor of the chest wall, the soft tissue of lower extremities, paravertebral tissues, and retroperitoneum. ${ }^{3,6}$

Ewing's sarcoma is a rare disease comprising $4-6 \%$ of all primary bone tumors. ${ }^{2,4,7}$ Extraskeletal Ewing's sarcoma (EES) is rarer. The common sites of EES are the chest wall, paravertebral region, retroperitoneum, soft tissues of lower extremities, and gluteal region. Ewing's sarcoma of the head-and-neck region constitutes about $1-4 \%$ of EES cases. ${ }^{5,7}$ Sinonasal EES is extremely rare. Diagnosis and management of ES can be challenging, as it has to be differentiated from other tumors that share the features of undifferentiated small round cells such as non-keratinizing carcinoma, small cell carcinoma, and sinonasal undifferentiated carcinoma (SNUC). The diagnosis of these entities requires a histopathological examination, immunohistochemistry, and a cytogenetic analysis along with a CT scan and MRI of the paranasal sinuses. The treatment for ES of a sinonasal region with the involvement of the skull base requires craniofacial resection to be followed by chemotherapy and/or radiotherapy. ${ }^{3}$

We hereby report a case of EES of the sinonasal tract in a young male with extension to the skull base and dural involvement that
1,2,4,5 Department of ENT, Fortis Hospital, Mohali, Punjab, India

${ }^{3}$ Department of ENT, Lok Nayak Hospital, Delhi, India

Corresponding Author: Anuragini Gupta, Department of ENT, Fortis Hospital, Mohali, Punjab, India, Phone: +91 9999828161, e-mail: anuragini25@hotmail.com

How to cite this article: Gupta A, Gupta S, Chaudhary D, et al. Endoscopic Endonasal Management of Extraskeletal Sinonasal Ewing's Sarcoma. Clin Rhinol An Int J 2020;13(1):15-17.

Source of support: Nil

Conflict of interest: None

was surgically managed by endonasal endoscopy with adequate removal of the tumor mass. English literature does not mention this approach so far. The patient was subjected to postoperative $\mathrm{CT} / \mathrm{RT}$ and is on a regular follow-up for the last 2 years and is doing well.

\section{Case Description}

A 31-year-old male patient presented to ENT OPD with complaints of right-sided headache associated with nausea and vomiting, right nasal obstruction, a mass protruding from the right nostril and associated with occasional nasal bleed for a duration of 4 months with aggravation of symptoms since last 15 days along with proptosis of the right eye and watering from right eye. Nasal examination revealed a reddish-brown friable slough covered mass protruding from the right nostril bleeding on manipulation. The left side nasal cavity was normal except for deviation of the septum to left. There was associated proptosis of the right eye with lateral protrusion of the eyeball with normal vision and no restriction of extraocular movements in any quadrants. Contrastenhanced CT scan and contrast-enhanced MRI paranasal sinuses were done which were suggestive of heterogeneously enhancing mass lesion in the bilateral anterior ethmoid sinus, right frontal 
sinus, erosion of floor of the anterior cranial fossa, superomedial and medial wall of the right orbit, and extension in extraconal fat (Fig. 1).

The patient also gave a history of biopsy done from nasal mass outside which was suggestive of sinonasal round cell tumor. The specimen slides and blocks were reviewed and were found to be suggestive of round cell tumor/PNET. On performing immunohistochemistry, it was found to be positive for CD99 marker which helped us to arrive at the diagnosis of ES (Figs 2 and 3).

After all relevant investigations and clearances patient was taken up for endoscopic endonasal excision of the mass lesion. Intraoperatively, unhealthy polypoidal mass was seen filling bilateral maxillary, ethmoid, sphenoid, and frontal sinus, destruction of the cartilaginous part of the nasal septum was found. Tumor mass was found eroding the right fovea ethmoidalis. Using endoscopic endonasal approach, tumor mass excised completely and sent for biopsy and skull base defect repaired using composite graft comprising of muscle and tensor fascia lata harvested from the thigh. The excised specimen sent for histopathological examination showed fragments of the nasal mucosa with an infiltrating neoplasm composed of small round cells arranged in sheets were seen suggestive of round cell tumor-ES/PNET which on further immunohistochemistry was strongly positive for CD99 marker. Molecular studies using PCR
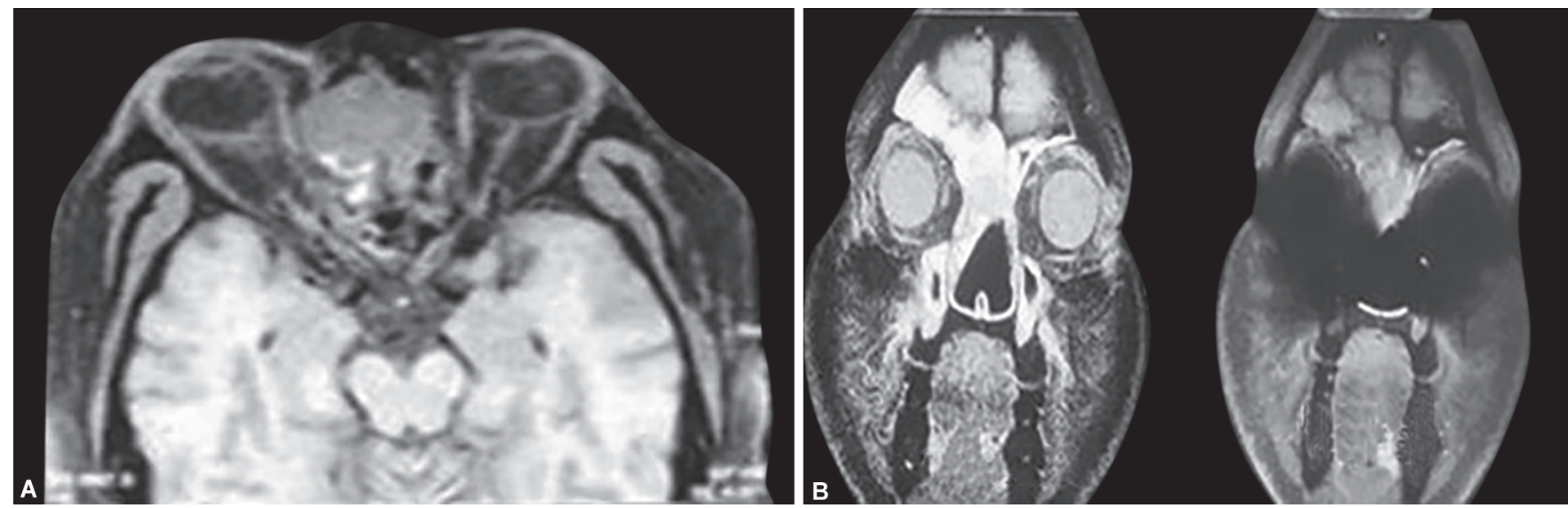

Figs $1 \mathrm{~A}$ and B: Preoperative radiological picture showing the extent of involvement by the tumor
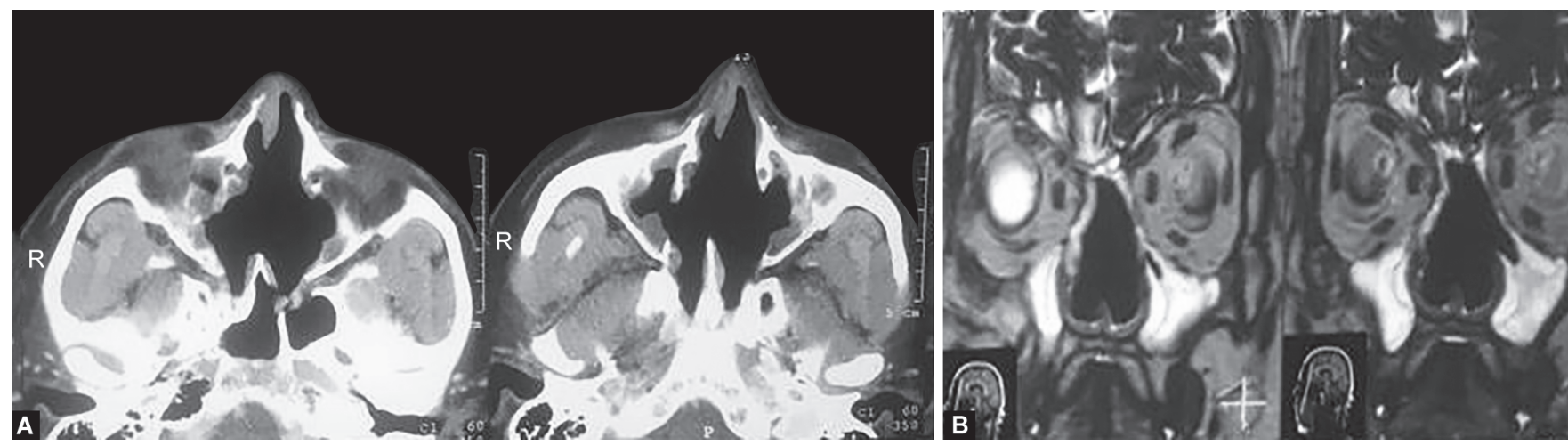

Figs $2 \mathrm{~A}$ and $\mathrm{B}$ : Postoperative radiological imaging
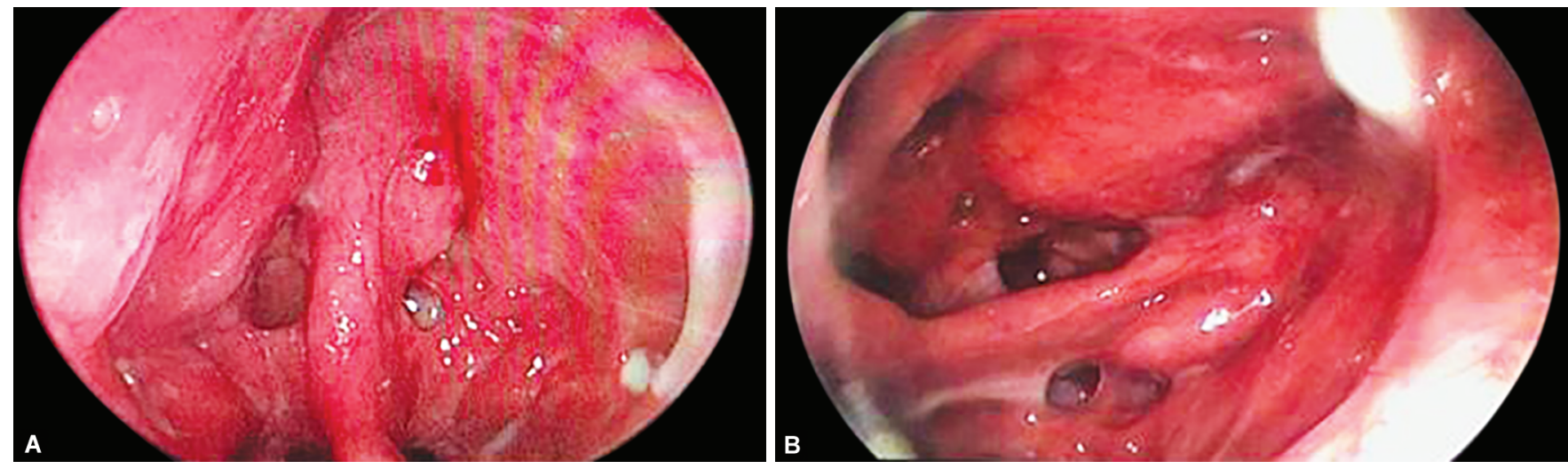

Figs $3 \mathrm{~A}$ and B: Postoperative nasal endoscopy at 2-year follow-up 
confirmed the chromosomal translocation of FLI1 (exon 6), hence proving the diagnosis of ES.

The patient underwent postoperative radiotherapy, received 28 fractions of 55 Gy radiations, and was added on 3 cycles of chemotherapy after completion of 21 fractions (vincristine, adriamycin, and cyclophosphamide). He completed his treatment and is on regular follow-up, 2 years postoperatively patient is asymptomatic with no signs of metastasis or recurrence.

\section{Discussion}

Ewing's sarcoma is a highly malignant, small, round cell tumor that originates from the primitive neuroectodermal cells. ${ }^{7}$ It was first described by James Ewing in $1921 .^{8}$ Extraskeletal Ewing's sarcoma is a rare, rapidly growing, round cell malignant tumor that can develop in the soft tissue at any location. Primary ES of the head and neck is uncommon. Among bone ES, the head-and-neck (skull) accounts for $3.8 \%$ of cases. Primary sinonasal ES is even rarer and represents only a small subset of these head and neck cancers. In the sinonasal tract, the various differential diagnosis of small round cell tumors is rhabdomyosarcoma, lymphoma, poorly differentiated carcinomas, melanoma, olfactory neuroblastoma (ONB), and ESFT. Among these tumors, ESFTs are rare in this location and have not been extensively reported in the literature. Primary ES commonly occurs in early childhood or adolescence and rarely in adulthood. There is a slightly male predominance with a male-to-female ratio of 1.5:1. 1,4,5 Patients with ES of the head-and-neck region less frequently have metastases at diagnosis. Microscopically, these tumors are composed of uniform small round cells with round nuclei containing fine chromatin and scant clear or eosinophilic cytoplasm. $^{3}$ The diagnostic battery includes histopathological examination, immunohistochemistry, and cytogenetic analysis. Immunohistochemistry is the essential diagnostic examination to differentiate Ewing sarcoma (EWS) from the many small round neoplasms is the CD99 marker. For the definitive diagnosis, molecular studies using PCR to detect the characteristics of chromosomal translocation can be done. The gene sequence, $t(11 ; 22)(q 24 ; q 12)$, which results in the fusion of the EWS gene with the FLI1 (exon 6) gene, is the genetic hallmark of ES.

Patients with sinonasal ES usually present with symptoms of nasal obstruction and epistaxis. ${ }^{5,9}$ A contrast-enhanced CT scan is used in the radiological evaluation of sinonasal ES, which shows an enhancing soft tissue mass with bone destruction. Usually, the ES shows a characteristic radiographic picture described by some authors as "onion skin appearance" especially in the long bones, but such a pattern is less commonly seen in the sinuses.

The effective treatment for ES includes combined surgical excision and chemotherapy/radiotherapy which have increased the 5-year survival rate. Due to advances in the treatment of ES in recent years, survival rates are reported to have improved and have reached up to $86 \%$ in patients with non-metastatic disease at initial presentation. ${ }^{1-3}$ Those with metastases have poorer outcomes.

In ES of the extremities, radical surgical resection (with or without radiotherapy) is superior to radiotherapy alone. However, there are literature reporting radical excision of the tumor via open surgical approaches, we report this case of endoscopic endonasal approach to excision of the tumor. In our case, the endoscopic endonasal approach with adequate tumor excision followed by postoperative combined chemotherapy and radiotherapy has achieved a good local control without the need for any further surgical intervention. The patient remains alive without evidence of recurrence or metastasis at 3 years since the first examination.

\section{Conclusion}

Ewing's sarcoma rarely affects the sinonasal tract. Treatment includes a multidisciplinary approach with surgery as the first line followed by chemotherapy and radiotherapy. A conservative endoscopic endonasal approach can achieve good local control when combined with adjuvant radiotherapy and chemotherapy. Ewing's sarcoma in the head-and-neck region does not metastasize early, hence carries a better prognosis.

\section{References}

1. Howarth K, Khodaei I, Karkanevatos A, et al. A sinonasal primary Ewing's sarcoma. Int J Pediatr Otorhinolaryngol 2004;68(2):221-224. DOI: 10.1016/j.ijporl.2003.09.011.

2. Hafezi S, Seethala RR, Stelow EB, et al. Ewing's family of tumors of the sinonasal tract and maxillary bone. Head Neck Pathology 2010;5(1):8-16. DOI: 10.1007/s12105-010-0227-x.

3. Yeshvanth S, Ninan K, Bhandary S, et al. Rare case of extraskeletal ewings sarcoma of the sinonasal tract. J Cancer Res Ther 2012;8(1):142144. DOI: $10.4103 / 0973-1482.95197$.

4. Balamuth NJ, Womer RB. Ewing's sarcoma. Lancet Oncol 2010;11(2):184-192. DOI: 10.1016/S1470-2045(09)70286-4.

5. Souheil J, Skander K, Sawssen D, et al. Ewing sarcomas of the sinonasal tract and maxillary bone. Egyptian J Ear Nose Throat Allied Sci 2016;17(3):147-153. DOI: 10.1016/j.ejenta.2016.08.001.

6. Negru ME, Sponghini AP, Rondonotti D, et al. Primary Ewings sarcoma of the sinonasal tract, eroding the ethmoid and sphenoid sinus with intracranial extension: a rare case report. Mol Clin Oncol 2015;3(4):807-810. DOI: 10.3892/mco.2015.548.

7. Lin JK. Sinonasal Ewing sarcoma: a case report and literature review. Perm J 2018;22:17-086.

8. Suzuki T, Yasumatsu R, Nakashima T, et al. Primary Ewing's sarcoma of the sinonasal tract: a case report. Case Rep Oncol 2017;10(1):91-97. DOI: 10.1159/000455040.

9. Vaccani J, Forte V, De Jong A, et al. Ewing's sarcoma of the head and neck in children. Int J Pediatr Otorhinolaryngol 1999;48(3):209-216. DOI: 10.1016/S0165-5876(99)00030-0. 